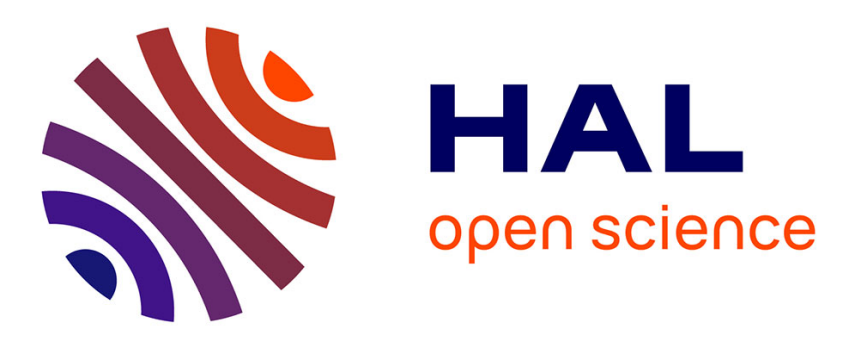

\title{
Elastically driven cooperative response of a molecular material impacted by a laser pulse
}

Roman Bertoni, Maciej Lorenc, Hervé Cailleau, Antoine Tissot, Jérôme

Laisney, Marie-Laure Boillot, Laurentiu Stoleriu, Alexandru Stancu, Cristian Enachescu, Eric Collet

\section{To cite this version:}

Roman Bertoni, Maciej Lorenc, Hervé Cailleau, Antoine Tissot, Jérôme Laisney, et al.. Elastically driven cooperative response of a molecular material impacted by a laser pulse. Nature Materials, 2016, 15 (6), pp.606-610. 10.1038/nmat4606 . hal-01294246

\section{HAL Id: hal-01294246 \\ https://hal.science/hal-01294246}

Submitted on 28 Mar 2016

HAL is a multi-disciplinary open access archive for the deposit and dissemination of scientific research documents, whether they are published or not. The documents may come from teaching and research institutions in France or abroad, or from public or private research centers.
L'archive ouverte pluridisciplinaire HAL, est destinée au dépôt et à la diffusion de documents scientifiques de niveau recherche, publiés ou non, émanant des établissements d'enseignement et de recherche français ou étrangers, des laboratoires publics ou privés. 


\title{
Elastically driven cooperative response of a molecular material impacted by a laser pulse
}

\author{
Roman Bertoni ${ }^{1}$, Maciej Lorenc ${ }^{1, *}$, Hervé Cailleau ${ }^{1}$, Antoine Tissot ${ }^{2, \dagger}$, Jérôme Laisney, ${ }^{2}$ Marie-Laure \\ Boillot $^{2}$, Laurentiu Stoleriu ${ }^{3}$, Alexandru Stancu ${ }^{3}$, Cristian Enachescu ${ }^{3}$, Eric Collet $^{1 *}$ \\ ${ }^{1}$ Université de Rennes1, Institut de Physique de Rennes, UMR UR1-CNRS 6251, F-35000 Rennes, France. \\ ${ }^{2}$ Institut de Chimie Moléculaire et des Matériaux d'Orsay,UMR-CNRS 8182, Université Paris-Sud, Orsay, France. \\ ${ }^{3}$ Faculty of Physics, Alexandru Ioan Cuza University, 700506, Iasi, Romania. \\ ${ }^{\dagger}$ Present address: Institut Lavoisier de Versailles, UMR 8180,Université de Versailles, Saint-Quentin en Yvelines, 45 Avenue des \\ Etats-Unis, 78035 Versailles Cedex, France.
}

*e-mail: eric.collet@univ-rennes1.fr.andmaciej.lorenc@univ-rennes1.fr

Photoinduced phase transformations ${ }^{[1,2]}$ occur when a laser pulse impacts a material, thereby transforming its electronic and/or structural orders, consequently directing the functionalities ${ }^{[3,4,5,6,7]}$. The transient nature of photoinduced states has thus far severely limited the application scope. It is of paramount importance to explore whether structural feedback during the solid deformation has capacity to amplify and stabilize photoinduced transformations. Contrary to coherent optical phonons long under scrutiny ${ }^{[8,9,10]}$, coherently propagating cell deformations over acoustic time-scale ${ }^{[11,12,13,14]}$ have not been explored to similar degree, particularly in light of cooperative elastic interactions. Herein we demonstrate experimentally and theoretically a self-amplified responsiveness in a spin-crossover material ${ }^{[15]}$ during its delayed volume expansion. The cooperative response at material scale prevails above a threshold excitation, significantly extending the lifetime of photoinduced states. Such elastically-driven cooperativity triggered by a light pulse offers a new efficient route to the generation and stabilization of photoinduced phases in many volumechanging materials.

To address the issue of the photoswitching of materials from the perspective of elastic cooperativity, notwithstanding its common perception related to electrons and optical phonons, we choose to study a system in which the elastic field has capacity to drive a phase transition. Among vast families of volume-changing materials, spin-crossover $(\mathrm{SCO})^{[15,16]}$ crystals are arguably best prototypes. Their switching occurs between low spin (LS) and high spin (HS) molecular states with different molecular volume. Many of these materials exhibit isostructural transitions with a significant change of crystalline volume accompanying the evolution of the fraction of $\mathrm{HS}$ molecules $\left(\mathrm{X}_{\mathrm{HS}}\right)$. The molecular photoswitching of SCO molecules from LS to HS states, induced by a femtosecond laser pulse, occurs within a few 100s fs, irrespective of the environment, as observed in densely packed SCO crystals ${ }^{[10,17]}$ and isolated molecules in solutions ${ }^{[18]}$. However, only in solids this instantaneous process triggers a subsequent transformation dynamics manifested by a step-like evolution of $\mathrm{X}_{\mathrm{HS}}$ in the time domain ${ }^{[13,14,17,19]}$. The crystal deformations, and volume change in particular, do not occur during the initial step. Indeed, homogeneous dilation requires homothetic translation of atoms over long distances. Thus defined elastic step is associated with the change of volume and involves propagation of strain waves. It takes place on the so-called acoustic time scale, determined by the ratio between the relevant length scale (penetration depth, laser beam size or crystal size) and the sound velocity in the medium. The coupling between the strain wave and the fraction of switched molecule raises a challenging question whether such coupling can induce a self-amplified transformation of the material.

For the $\left[\mathrm{Fe}^{\mathrm{III}}(3-\mathrm{MeO}-\mathrm{SalEen})\right] \mathrm{PF}_{6}$ material investigated here, the molecules swell and the crystal volume expands by $3 \%$ during the thermal transition from $\operatorname{LS}(\mathrm{S}=1 / 2)$ to $\mathrm{HS}$ $(\mathrm{S}=5 / 2)$ states $^{[20]}$. We use time-resolved optical spectroscopy to study cooperative elastic effects in this SCO material (see methods). Samples were photo-excited at $140 \mathrm{~K}$ in the LS phase by a $800 \mathrm{~nm}$ femtosecond laser pulse, efficiently switching molecules from $\mathrm{LS}$ to $\mathrm{HS}^{[17,20]}$. In order to validate the rationale of the acoustic time scale we study samples of very different size: single-crystals $(1)[(300 \pm 50) \times(200 \pm 50)$ $\left.\times(15 \pm 5) \mu^{3}\right]$, micro-crystals $(2)[(7.5 \pm 2.1) \times(0.64 \pm 0.15) \times$ $\left.(0.21 \pm 0.03) \mu \mathrm{m}^{3}\right]$ and nano-crystals (3) $[(950 \pm 150) \times$ $\left.(270 \pm 40) \times(35 \pm 7) \mathrm{nm}^{3}\right],(2)$ and (3) being dispersed in PVP (polyvinylpyrrolidone) thin films.

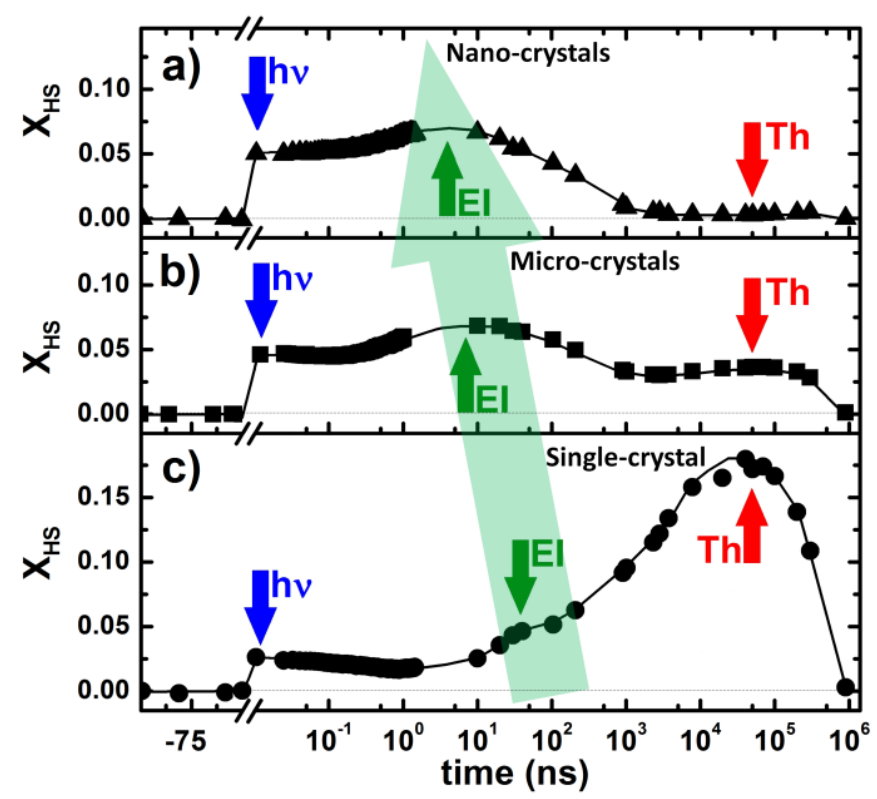

Figure 1 Response to femtosecond laser excitation of LS [Fe"II-MeO-SalEen)]PF 6 in the form of nano-crystals (a), micro-crystals (b) and single-crystal (c), black lines are guide to the eye. "hv", "El" and "Th" denote respectively the photoinduced, elastic and thermal steps. The green arrow underlines the shift of the elastic step towards shorter time as the size of the system is reduced. 

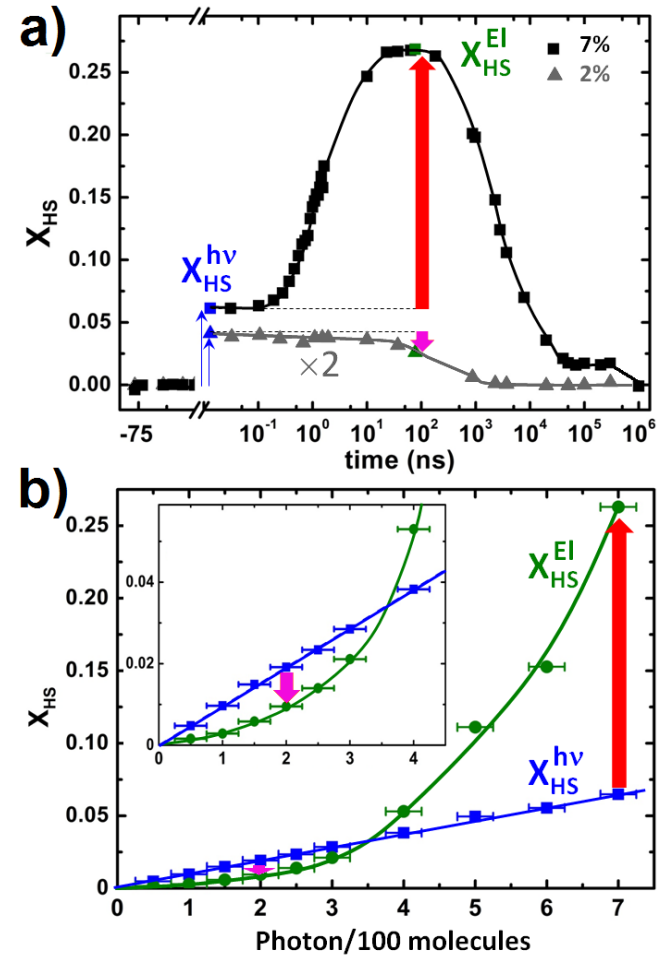

Figure 2 Photoresponse of nano-crystals to excitation density. a) Time course of $X_{H S}$ following femtosecond laser excitation for $7 \%$ (black) and $2 \%$ (grey, scaled $\times 2$ ) of photoexcited molecules (lines are guide to the eye). The difference between $X_{H S}{ }^{h_{V}}$ (dotted horizontal line) and $X_{H S}{ }^{E I}$, corresponds to relaxation (pink down arrow) or amplification (red up arrow). b) Linear evolution of $X_{H S}{ }^{h_{\nu}}$ (measured at 10 ps) and non-linear evolution of $X_{H S}{ }^{E I}$ (measured at $100 \mathrm{~ns}$ ) with laser excitation density (with error bars) scaled in photon/100 molecules.

The time evolution of $\mathrm{X}_{\mathrm{HS}}$ after fs laser excitation of (1), (2) and (3) shown in Fig. 1a-c was monitored by optical density change over several time decades from fs to ms (see methods). The 3-step response of these crystals is essentially similar to that reported recently for other less cooperative SCO materials ${ }^{[13,14]}$. At first, a fraction $X_{H S}{ }^{h v}$ of molecules photoswitches locally from LS to HS via ultrafast intersystem crossing, accompanied by a molecular swelling within $100 \mathrm{~s}$ $\mathrm{fs}^{[10,17]} \cdot X_{H S}{ }^{h v}$ increases with downsizing the samples below the laser penetration length $(\approx 5 \mu \mathrm{m}$ at $800 \mathrm{~nm})$ allowing a homogeneous excitation in micro-crystals and nano-crystals. During this initial step of transformation the crystal does not exchange energy with its environment. A second increase of the HS fraction, yielding $X_{H S}^{E l}$, occurs upon the elastic step in the 0.1-50 ns range (green arrows in Fig. 1). The elastic step proceeds with the establishment of mechanical equilibrium with the environment. Time-resolved x-ray diffraction on single crystals ${ }^{[13,14]}$ characterized directly the cell volume expansion and revealed a strain waves propagation at a speed similar to acoustic waves (measured to be $\sim 2000 \mathrm{~m} . \mathrm{s}^{-1}$ ). ${ }^{[21]}$ This volume expansion occurs well after the initial photoswitching. The observed shift of this step by two temporal decades $(20 \mathrm{~ns}$ for single-crystals, $1 \mathrm{~ns}$ for microcrystals and 300 ps for nano-crystals) agrees with the crystal size reduction ratio. The lattice expansion results from change in internal pressure, due to two effects: ( $i$ ) the swelling of photoswitched HS molecules forming in an initially constrained LS lattice, and (ii) the lattice heating due to energy dissipation into the phonon bath ${ }^{[13,14]}$. This energy redistribution leads to a global temperature rise of the crystal, a)
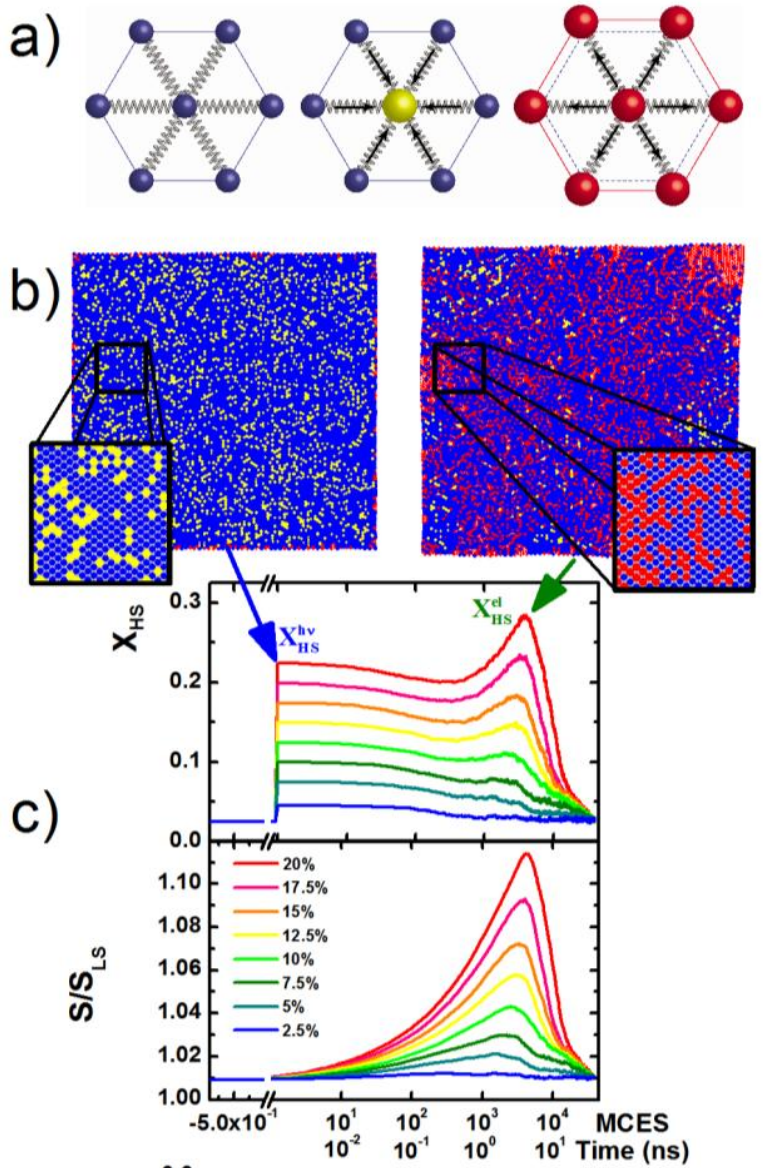

d)

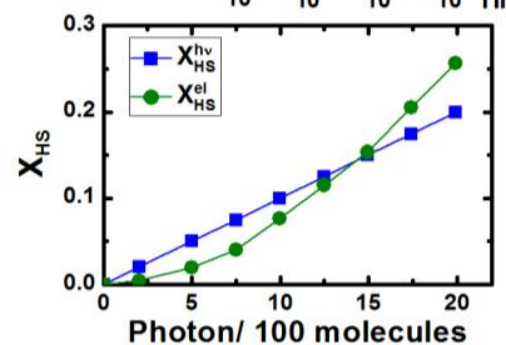

Figure 3 Monte Carlo simulations with the mechanoelastic model. a) LS (blue) and HS (red) molecules in their close environment. In a LS lattice the HS molecules feel a high pressure (yellow). b) Snapshots of a system composed of 13826 molecules just after random switching of molecules (left) and after the lattice expansion (right), molecular states are color coded as in a). c) Time course in MCES (scaled to ns) of HS fraction and lattice surface normalised to the LS state for different percentage of photoexcitation. d) Evolution of the HS fraction on the photoswitching and elastic steps as a function of the initial fraction of excited molecules.

hence the third so-called thermal step, upon which the HS population thermally equilibrates at $X_{H S}{ }^{T h}$ level. The thermal equilibration timescale $(\approx 50 \mu \mathrm{s}$, red arrows) depends on the local molecular energy barrier between LS and HS states, and remains sample size independent ${ }^{[17]}$. The thermal population is large for an isolated single-crystal but becomes smaller for micro- and nano-crystals dispersed in PVP, as their surface/volume ratio allows for efficient heat transfer to the polymer, a heat sink limiting the thermal population of HS state. In the following, we focus our attention on the nanocrystals (3), for which molecular switching occurs predominantly upon the elastic step and remains negligible upon heating. 
Figure 4 schematics of the mechanism:

a) at low excitation, few molecules are photoswitched and recover the LS state (blue) under the effect of the pushing lattice (inset, blue arrows). b) at high excitation the lattice expands and pulls (inset, red arrows) LS molecules, which switch cooperatively to the HS state (red) of higher molecular volume.

Fig. 2a shows the time evolution of $X_{H S}$ in nanocrystals over the complete course of the out-of-equilibrium dynamics, under two regimes of excitation density. Fig. 2b shows that $X_{H S}^{h v}$, a) low excitation

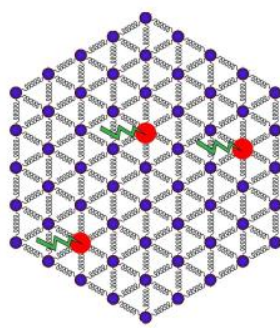

b) high excitation measured at the picosecond time scale, depends linearly on the excitation density, thereby manifesting the local and non cooperative nature of the photoswitching process. Since the $\mathrm{LS} \rightarrow \mathrm{HS}$ photoswitching in these materials exhibits a quantum efficiency close to $1^{[10,17]}$, we scale the laser excitation density in photon $/ 100$ molecules. When the fraction of photoswitched molecules from LS to HS state is low $\left(X_{H S}{ }^{h v}=2 \%\right)$, the LS ground state is recovered within 1 $\mu \mathrm{s}$. At higher excitation density $\left(X_{H S}{ }^{h v}=7 \%\right) X_{H S}{ }^{E l}$ increases, up to fivefold during the elastic step (see also Fig. S1 \& S2). It is a clear manifestation of non-linear underpinnings of the process leading to self-amplified response on the acoustic time-scale, following light excitation. The evolution of $X_{H S}{ }^{E l}$ with the excitation density (Fig. 2b) reveals a pronounced threshold whereby the system response clearly departs from linear regime. For excitation below $\approx 3.5 \%$ the photoswitched molecules relax and $X_{H S}{ }^{E l}<X_{H S}^{h v}$, whereas above this threshold a self-amplification occurs and $X_{H S}{ }^{E l}>X_{H S}{ }^{h v}$. Such non-linear and self-amplified response on the elastic step above the threshold excitation, also observed on single crystals (Fig. S3), evidences the active role of the crystalline medium and the cooperative nature of this process.

These experimental observations, providing a qualitative rationale, are corroborated by an extension of the recently developed mechanoelastic (ME) model ${ }^{[22,23,24]}$. In this balland-spring model (Fig. 3a), the elastic interactions arise from lattice distortions due to the difference of molecular volumes between the LS and HS states. The SCO molecules are represented as rigid spheres, larger in HS state, arranged in open boundary lattices and interacting by the way of connecting springs, with the elastic constant $k$. The volume change of a switched molecule exerts an instantaneous elastic force on its neighbouring springs and consequently determines the shift in initial position of its closest neighbours. Because of the molecule-to-molecule interactions every molecular switch modifies the volume and the shape of the whole crystal. For the simulations, we use a rectangular shape sample, composed of $13924(\approx 118 \times 118)$ molecules in a triangular configuration (Fig. 3b). Such number of molecules is large enough to reproduce properties of the spin crossover system and to avoid false local minima in dynamical simulations. ${ }^{[20,22]}$ besides approaching the number of molecules in nano-crystals (there are $\approx 300$
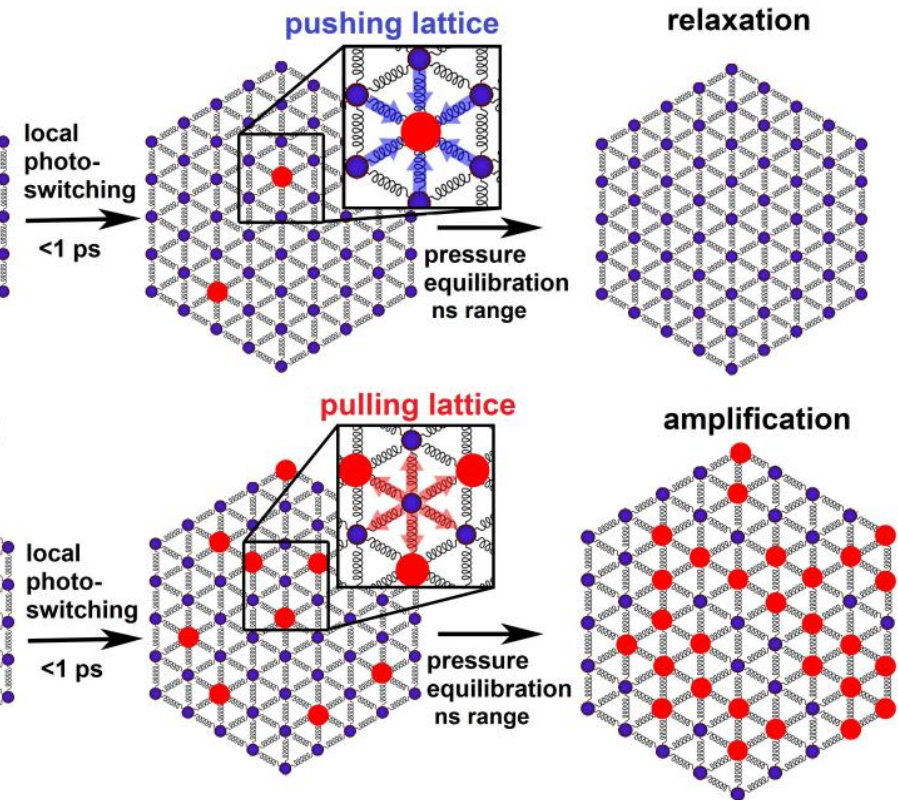

molecules along a typical size of $\approx 300 \mathrm{~nm}$ ). The different parameters of the model $(\Delta \mathrm{H}=1100 \mathrm{~K}$ and $\Delta \mathrm{S}=7)$ were chosen to reproduce the $\approx 157 \mathrm{~K}$ thermal conversion temperature, see also supplementary information. We consider a temperature at which most of the molecules are equilibrated in the LS state $(\mathrm{T}=145 \mathrm{~K})$. For reproducing the laser pulse effect, a homogenous photoexcitation is emulated by choosing randomly at $\mathrm{MCES}=1$ a fraction of $\mathrm{HS}$ molecules and by increasing their temperature by $\Delta T=100$ $\mathrm{K}$. The system evolves and the switched molecules equilibrate thermally with the lattice (3000 MC steps). The resulting lattice heating depends on the fraction of excited molecules ( $2 \mathrm{~K}$ for $2 \%$ and $20 \mathrm{~K}$ for $20 \%$ ). These values are of the same order as the $5-30 \mathrm{~K}$ lattice heating experimentally observed $^{[13,14]}$.

With this model, we simulate in Fig. 3c the time course in MCES (scaled to real time as explained in Supp. Mat.) of $X_{H S}$ and the crystal volume (surface in our 2D model) for different photoexcitation levels, considering that one photon switches a single molecule on the photoswitching step. When $X_{H S}^{h v}$ is high (above 15\%) the HS fraction starts increasing around 1000 MCES, up to a maximum $X_{H S}{ }^{E l}$ reached around 4000 MCES, concomitantly with the maximum surface expansion. This incubation time reproduces well that observed experimentally for the onset of self-amplification. The changes inside the lattice take certain time to propagate towards the edges of the crystal and the surface variation does not follow the instantaneous increase of $X_{H S}$ at MCES step 1 . When $X_{H S}{ }^{h v}$ is low (2\%) the HS fraction monotonously decreases and the lattice expansion on the elastic step is weak, hence the relaxation with $X_{H S}^{E l}<X_{H S}^{h v}$ is well reproduced too. The non-linear response at the elastic step (Fig. 2) with the fraction of photoexcited molecules is reliably reproduced here (Fig. 3c \& d). The mapping of the system reveals that immediately after the photoexcitation a large number of molecules (yellow) are affected by high pressure. This local pressure on the photoswitched molecules equilibrates during the elastic step, which is mostly due to the development of regions (located towards corners or edges), where the local energetic conditions (or local pressure) favour the HS state. Such hypothesis is supported by Fig. 3b showing snapshots of the system just after the photoexcitation and after the elastic step. We should also underline that the mechanoelastic model uses harmonic 
potential devoid of thermal expansion. In other words, only the increase of the fraction of HS molecules of larger volume is responsible for lattice expansion, not its heating. The elastic nature of this process is also confirmed by the fact that a similar self-amplification is successfully simulated without taking into account lattice heating (Fig. S4) and occurs on longer timescales if the size of the system increases (Fig. $1 \& S 5)$.

Both experimental and theoretical analyses reveal another type of cooperative phenomenon triggered by light. Our study unambiguously relates the self-amplified molecular transformation to the macroscopic volume expansion during the elastic step. Fig. 4 depicts the mechanism. A fraction of molecules, $X_{H S}^{h v}$, is initially photoswitched from LS to HS and they locally swell within $100 \mathrm{~s}^{\mathrm{fs}}{ }^{[10,17]}$. These larger HS molecules are pushed back by forces applied by predominantly LS lattice, since its volume is fixed on short timescale (Fig. 4a). When $X_{H S}{ }^{h v}$ is low, photoswitched HS molecules rapidly relax to the elastically favoured LS state. Reversely, photoswitched molecules trigger lattice expansion over large scale in space. When $X_{H S}^{h v}$ is high enough the propagating lattice expansion, which re-establishes mechanical equilibrium, becomes sufficient to pull LS molecules (Fig. 4b) to HS state before the relaxation of photoswitched molecules. Reminiscent of a feedback mechanism intrinsic to active media, the more volume expands, the more molecules switch, the more volume expands, ... This simple physical picture is based on the competition between the pushing forces on HS molecules and the pulling forces on LS molecules. Since the latter increases with the fraction of switched molecules these competing forces set the underpinnings of the non-linear response to photoexcitation. Hence, the elastically-driven response resulting from the simultaneous absorption of many photons is greater than the sum over individual responses of constituting molecules, which is the direct manifestation of cooperative effects induced by light pulse. Due to the long range nature of elastic interactions, the final distribution of switched molecules remains essentially homogeneous in space, except near edges where mechanical forces are different. The above mechanism is fundamentally different from thermally activated much slower (sec-min) nucleation and domains growing ${ }^{[25]}$.

The revealed elastically-driven cooperativity activated by light pulse far exceeds the action of photons, leading at best to single molecular switching. In the system discussed herein, more than 5 molecules per photon are switched above the cooperativity threshold, on a time scale 4 orders of magnitude faster than thermal effects. This elastic effect is very likely responsible for non-volatile switching inside thermal hysteresis ${ }^{[26]}$. Elastic coupling between SCO molecules was a long-purported mechanism responsible for self-accelerated LIESST relaxation ${ }^{[26,27]}$. Our finding and proposed rational, underpinned by the very same elastic coupling, is the experimental demonstration of the opposite mechanism leading to self-amplified LIESST transformation. The proposed concept of coupling between the strain wave and the order parameter field should be valid for many, if not all, volume-changing materials of which phase transitions reveal significant crystal deformations, such as breathing crystals or Mott-insulators to name a few ${ }^{[29,30,31,32,33,34]}$. In many material the initial photo-process is local, but it may exert pulling or pushing forces on the crystalline medium, and trigger similar elastic cooperative response stabilizing new phase. This may help circumvent the severe limitation imposed by the transient nature of photoinduced transformations observed in a vast majority of photoinduced phenomena, where the fast relaxation of excited electronic state and rearrangements of atoms inside unit cell typically take place before the elastic step. Here HS molecules in a rigid LS lattice decay with a $\approx 1$ ns time constant, as observed in single crystals (Fig. 1). But in nanocrystals the volume expansion proceeds before complete molecular relaxation. Consequently, the amplification takes over the relaxation on sub-ns time scale, which is 4 orders of magnitude faster than the onset of thermal effects that may reside in a system prone to thermal conversion. Our 2D hexagonal model finds a threshold when 1 molecule out of 7 is excited. Our data on $3 \mathrm{D}$ crystal points a threshold close to $3.5 \%$ i.e. one excited molecule in a cell of $3 \times 3 \times 3$. Both results indicate that the amplification starts with sufficient spatial correlation, when an average molecule is connected to one nearest-neighbour excited molecule (Fig. 3b).

The recent observation of photo-reversible switching triggered by a ns pulse in a nanomaterial of metal oxide ${ }^{[29]}$ corroborates our findings. Such issues are of paramount importance both from the fundamental stand point, and for the light-control of non-volatile information.

Supplementary Information is linked to the online version of the paper at www.nature.com/nmat.

\section{References}

1 Nasu, K. Photoinduced Phase Transitions. (World Scientific, 2004).

2 Cailleau, H., Lorenc, M., Buron-Le Cointe, M., Servol, M., Cammarata, M., Collet E. Impacting materials by light and seeing their structural dynamics. Eur. Phys. J. 222, 1077-1092 (2013).

3 Ichikawa, H. Nozawa, S., Sato, T., Tomita, A., Ichiyanagi, K., Chollet, M. Guerin, L., Dean, N., Cavalleri, A., Adachi, S., Arima, T., Sawa, H., Ogimoto, Y., Nakamura, M., Tamaki, R., Miyano, K., Koshihara, S. Transient photoinduced 'hidden' phase in a manganite. Nature Mater. 10, 101-105 (2011)

4 Gao, M., Lu, C., Jean-Ruel, H., Liu, L. C., Marx, A., Onda, K., Koshihara, S. Nakano,Y., Shao, X., Hiramatsu, T., Saito, G., Yamochi, H., Cooney, R. R., Moriena, G., Sciaini, G. \& Miller, R.J.D. Mapping molecular motions leading to charge delocalization with ultrabright electrons. Nature 496, 343-346 (2013).

5 Porer, M., Leierseder, U., Ménard, J.-M., Dachraoui, H., Mouchliadis, L., Perakis, I. E., Heinzmann, U., Demsar, J., Rossnagel, K., Huber, R. Non-thermal separation of electronic and structural orders in a persisting charge density wave. Nature Mater. 13, 857-861 (2014)

6 Beaud, P., Caviezel, A., Mariager, S. O., Rettig, L., Ingold, G., Dornes, C., Huang, S-W., Johnson, J. A., Radovic, M., Huber, T., Kubacka, T., Ferrer, A., Lemke, H. T., Chollet, M., Zhu, D., Glownia, J. M., Sikorski, M., Robert, A., Wadati, H., Nakamura, M., Kawasaki, M., Tokura, Y., Johnson, S. L., Staub U. A time-dependent order parameter for ultrafast photoinduced phase transitions. Nature Mater. 13, 923-927 (2014)

7 Collet, E., Lemée-Cailleau, M.-H., Buron-Le Cointe, M., Cailleau, H., Wulff, M. Luty, T., Koshihara, S., Meyer, M., Toupet, L., Rabiller, P., Techert, S. Laserinduced ferroelectric structural order in an organic charge-transfer crystal. Science 300, 612-615 (2003).

8 Sokolowski-Tinten, K., Blome, C., Blums, J., Cavalleri, A., Dietrich, C., Tarasevitch, A., Uschmann, I., Förster, E. Kammler, M., Horn-von-Hoegen, M., von der Linde D. Femtosecond X-ray measurement of coherent lattice vibrations near the Lindemann stability limit. Nature 422, 287-289 (2003).

9 Wall, S., Wegkamp, D., Foglia, L., Appavoo, K., Nag, J., Haglund Jr, R.F., Stähler, J. M., Wolf. Ultrafast changes in lattice symmetry probed by coherent phonons, Nature Com 3, 721 ( 2012).

10 M. Cammarata, R. Bertoni, M. Lorenc, H. Cailleau, S. Di Matteo, C. Mauriac, S. F. Matar, H. Lemke, M. Chollet, S. Ravy, C. Laulhé, J.-F. Létard and E. Collet. Sequential activation of molecular breathing and bending during spin-crossover photoswitching revealed by femtosecond optical and X-ray absorption spectroscopy. Phys. Rev. Lett., 113, 227402 (2014).

11 Feurer, T., Vaughan, J.C., Nelson, K.A. Spatiotemporal coherent control of lattice vibrational waves. Science 299, 374-377 (2003).

12 Lejman, M., Vaudel, G., Infante, I.C., Gemeiner, P., Gusev, V.E., Dkhil, B, Ruello, P. Giant ultrafast photo-induced shear strain in ferroelectric $\mathrm{BiFeO}_{3}$. Nature com. 5, 4301 (2014).

13 Lorenc, M., Balde, C., Kaszub, W., Tissot, A., Moisan, N., Servol, M., Buron-Le Cointe, M. Cailleau, H., Chasle, P., Czarnecki, P., Boillot, M.-L., Collet, E. Cascading photoinduced, elastic, and thermal switching of spin states triggered by a femtosecond laser pulse in an Fe(III) molecular crystal. Phys. Rev. B., 2012, 85, 054302 (2012)

14 Collet, E., Moisan, N., Baldé, C., Bertoni, R., Trzop, E., Laulhé, C., Lorenc, M. Servol, M., Cailleau, H., Tissot, A., Boillot, M.-L., Graber, T., Henning, R. Coppens, P. \& Buron-Le Cointe, M. Ultrafast spin-state photoswitching in a 
crystal and slower consecutive processes investigated by femtosecond optical spectroscopy and picosecond X-ray diffraction. Phys. Chem. Chem. Phys. 14, 6192-6199 (2012).

15 M. Halcrow, Ed., Spin-crossover materials (Wiley, West Sussex, 2013) ISBN 9781119998679

16 Willenbacher, N., Spiering, H. The elastic interaction of high-spin and low-spin complex molecules in spin-crossover compounds. J. Phys. C: Solid State Phys. 21, 1423 (1988)

17 Bertoni, R., Lorenc, M., Tissot, A., Boillot M.-L., Collet, E. Femtosecond photoswitching dynamics and microsecond thermal conversion driven by laser heating in $\mathrm{Fe}^{\mathrm{III}}$ spin-crossover solids. Coord. Chem. Rev., 2015, 282-283, 66-76 (2015)

18 Zhang, W., Alonso-Mori, R., Bergmann, U., Bressler, C., Chollet, M., Galler, A., Gawelda, W., Hadt, R. G., Hartsock, R. W., Kroll, T., Kjær, K. S., Kubiček, K., Lemke, H. T., Liang, H. W., Meyer, D. A., Nielsen, M. M., Purser, C., Robinson, J. S., Solomon, E. I., Sun, Z., Sokaras, D., van Driel, T. B., Vankó, G., Weng, T.C., Zhu, D., Gaffney, K.J. Tracking excited-state charge and spin dynamics in iron coordination complexes. Nature 509, 345-348 (2014)

19 Lorenc, M., Hébert, J., Moisan, N., Trzop, E., Servol, M., Buron-Le Cointe, M. Cailleau, H., Boillot, M.-L., Pontecorvo, E., Wulff, M., Koshihara, S., Collet E. Successive dynamical steps of photoinduced switching of a molecular Fe(III) spincrossover material by time-resolved x-ray diffraction. Phys. Rev. Lett. 103, 028301 (2009).

20 Tissot, A., Bertoni, R., Collet, E., Toupet, L., Boillot, M.-L. The cooperative spinstate transition of an iron(III) compound $\left[\mathrm{Fe}^{\mathrm{III}}(3-\mathrm{MeO}-\mathrm{SalEen})_{2}\right] \mathrm{PF}_{6}$ : thermal- vs ultra-fast photo-switching. J. Mat. Chem. 35, 2333-2340 (2011)

21 Félix, G., Mikolasek, M., Peng, H., Nicolazzi, W., Molnár, G., Chumakov, A.I., Salmon, L. Bousseksou, A. Lattice dynamics in spin-crossover nanoparticles through nuclear inelastic scattering. Phys. Rev. B 91, 024422 (2015)

22 Enachescu, C., Stoleriu, L., Stancu, A., Hauser, A. Model for Elastic Relaxation Phenomena in Finite 2D Hexagonal Molecular Lattices. Phys. Rev. Lett. 102, 257204 (2009).

23 Tissot, A., Enachescu, C., Boillot, M.-L. Control of the thermal hysteresis of the prototypal spin-transition $\mathrm{Fe}^{\mathrm{II}}(\text { phen })_{2}(\mathrm{NCS})_{2}$ compound via the microcrystallites environment: experiments and mechanoelastic model. J. Mater. Chem. 22, 20451 (2012)

24 Stoleriu, L., Chakraborty, P., Hauser, A., Stancu, A., Enachescu, C. Therma hysteresis in spin-crossover compounds studied within the mechanoelastic model and its potential application to nanoparticles. Phys. Rev. B 84, 134102 (2011)

25 Varret, F., Slimani, A., Boukheddaden, K., Chong, C., Mishra, H., Collet, E., Haasnootc, J., Pillet, S. The propagation of the thermal spin transition of $\left[\mathrm{Fe}(\mathrm{btr})_{2}(\mathrm{NCS})_{2}\right] \mathrm{H}_{2} \mathrm{O}$ single crystals, observed by optical microscopy. New J. Chem. 35, 2333-2340 (2011).

26 Cobo, S., Ostrovskii, D., Bonhommeau, S., Vendier, L., Molnar, G., Salmon, L., Tanaka, K., Bousseksou, A. Single-Laser-Shot-Induced Complete Bidirectional Spin Transition at Room Temperature in Single Crystals of (Fe ${ }^{\mathrm{II}}($ pyrazine $\left.)\left(\mathrm{Pt}(\mathrm{CN})_{4}\right)\right)$. J. Am. Chem. Soc. 130, 9019 (2008).

27 Hauser, A., Gütlich, P., Spiering, H. High-spin $\rightarrow$ low-spin relaxation kinetics and cooperative effects in the $\left[\mathrm{Fe}(\mathrm{ptz})_{6}\left(\mathrm{BF}_{4}\right)_{2}\left[\mathrm{Zn}_{1-\mathrm{x}} \mathrm{Fe}_{\mathrm{x}}(\mathrm{ptz})_{6}\right]\left(\mathrm{BF}_{4}\right)_{2} \quad(\mathrm{ptz}=1\right.$ Propyltetrazole) spin-crossover systems. Inorg. Chem. 25, 4245-4248 (1986).

28 Gütlich, P., Hauser, A., Spiering, H. Thermal and optical switching of Iron (II) complexes. Angew. Chem. Int. Ed. 33, 2024-2054 (1994)

29 Kobatake, S., Takami, S., Muto, H., Ishikawa, T. \& Irie, M. Rapid and reversible shape changes of molecular crystals on photoirradiation. Nature 446, 778-781 (2007).

30 Ohkoshi, S., Tsunobuchi, Y., Matsuda, T., Hashimoto, K., Namai, A., Hakoe, F. \& Tokoro H. Synthesis of a metal oxide with a room-temperature photoreversible phase transition. Nat. Chem. 2, 539-545 (2010).

31 Camjayi, A., Acha, C., Weht, R., Rodríguez, M. G., Corraze, B., Janod, E., Cario, L., Rozenberg, M. J. First-Order Insulator-to-Metal Mott Transition in the Paramagnetic 3D System $\mathrm{GaTa}_{4} \mathrm{Se}_{8}$. Phys. Rev. Lett. 113, 086404 (2014)

32 Veber, S. L., Fedin, M. V., Potapov, A. I., Maryunina, K. Yu., Romanenko, G. V., Sagdeev, R. Z., Ovcharenko, V. I., Goldfarb, D., Bagryanskaya, E. G. High-Field EPR Reveals the Strongly Temperature-Dependent Exchange Interaction in "Breathing" Crystals Cu(hfac)2LR. J. Am. Chem. Soc. 130, 2444-2445 (2008)
33 Okamoto, H., Ishige, Y., Tanaka, S., Kishida, H., Iwai, S., Tokura, Y. Photoinduced phase transition in tetrathiafulvalene-p-chloranil observed in femtosecond reflection spectroscopy. Phys. Rev. B 70, 165202 (2004).

34 Okimoto, Y., Peng, X., Tamura, M., Morita, T., Onda, K., Ishikawa, T., Koshihara, S., Todoroki, N., Kyomen, T., Itoh M.. Ultrasonic Propagation of a Metallic Domain in $\operatorname{Pr}_{0.5} \mathrm{Ca}_{0.5} \mathrm{CoO}_{3}$ Undergoing a Photoinduced Insulator-Metal Transition. Phys. Rev. Lett. 103, 027402 (2009).

Methods The evolution of the fraction of molecules in the HS state with the time delay after femtosecond laser excitation was obtained by transient optical density measurements. We used the optical pump-probe set-up described in ref[13], where the synchronization between the pump and probe femtosecond amplifiers is electronically tuned for $10 \mathrm{~ns}$-ms delays, while a mechanical translation stage sets the optical path difference for sub-ns measurements. The pump wavelength was set to $800 \mathrm{~nm}$ on a LMCT band where it efficiently induces LS-to-HS transition within 100s fs[17], below $140 \mathrm{~K}$ in the completely LS state. The probe wavelength was set to $550 \mathrm{~nm}$ where the optical density change between LS and HS is maximum. The time course of $X_{H S}(t)$ was determined by scaling the change of $\triangle O D(t)$, to the absolute change of OD between completely LS and HS states measured at thermal equilibrium.

\section{Author information:}

*Correspondence and requests for materials should be addressed to E.C. (eric.collet@univ-rennes1.fr.) and M.L. (maciej.lorenc@univ-rennes1.fr.).

\section{Acknowledgements}

This work was supported by the Institut Universitaire de France, Rennes Métropole, Région Bretagne (CREATE 4146), ANR (ANR-13-BS04-0002) and Europe (FEDER). CE thanks CNCS-UEFISCDI Romania (grant number PN-IIRU-TE-2014-4-0987).

\section{Author contributions:}

E.C. and M.L. conceived and coordinated the project. R.B. performed time-resolved optical measurements and analyzed the data. A.T., J.L. and M.-L. B. grew and prepared the samples. L. S., A. S. and C.E. extended the mechanoelastic model and performed Monte-Carlo simulations. E.C., H.C., M.L., R.B. and C.E. set the physical picture and wrote the paper. All authors contributed to discussions and gave comments on the manuscript.

\section{Competing financial interests}

The authors declare no competing financial interests 